\title{
A rare cause of acute urinary retention in a young man: A median raphe penile cyst
}

\author{
Mohd. Nazli Kamarulzaman, Azhani Chik, Hamid Ghazali \\ Kamarulzaman MN, Chik A, Ghazali H. A rare cause of acute urinary retention in a young man: A median raphe penile cyst. Malays Fam Physician. \\ 2021;16(1);114-116. https://doi.org/10.51866/cr1016
}

\section{Keywords:}

median raphe cyst of the

penis, acute urinary retention

\section{Authors:}

Mohd. Nazli Kamarulzaman

(Corresponding author)

Urology Unit, Department of Surgery

Kulliyyah of Medicine, International

Islamic University Malaysia

Kuantan, Pahang, Malaysia

Email:

*nazlikamarulzaman@gmail.com

\section{Azhani Bt Chik}

Department of Surgery, Department of Surgery, Kulliyyah of Medicine International Islamic University Malaysia, Kuantan, Pahang Malaysia

\section{Hamid Ghazali}

Department of Urology, Hospital Tengku Ampuan Afzan, Jalan Tanah Putih, Kuantan, Pahang, Malaysia

\section{Abstract}

Background: A penile median raphe cyst is an uncommon congenital lesion that is formed due to failure of the median raphe to close completely during embryo development. The majority of the cysts are asymptomatic and often go unnoticed. Here, we report acute urinary retention as a very rare complication of a penile median raphe cyst.

Case presentation: A 21-year-old single man presented with a sudden onset of acute urinary retention of one days' duration. Urethral catheterization was done at the Emergency Department and this drained $800 \mathrm{ml}$ of urine. On further questioning, he claimed that he had had a painless nodule at the glans penis since childhood. The swelling increased in size in the past week causing discomfort. He denied any history of genitalia trauma or recent sexual intercourse. On examination, there was a bluish lesion over the ventral aspect of the glans penis measuring about $3 \times 2 \mathrm{~cm}$. The lesion was later excised and histopathology revealed a median raphe cyst of the penis.

Conclusion: A penile median raphe cyst is a rare lesion. Acute urinary retention caused by this lesion is very rare.

\section{Introduction}

Congenital lesions in the genitalia area commonly present during early childhood. However, some can manifest later in adulthood and pose a diagnostic challenge to the clinician. Congenital median raphe cysts are relatively rare and are often ignored by the patient until they become complicated.

A median raphe cyst often presents as a small yellowish solitary papule lesion. ${ }^{1}$ Thus, it almost always goes unnoticed. At the time of presentation, this lesion commonly has some form of complications such as pain, increase in size, change in color, or difficulty in passing urine. ${ }^{1-2}$ Rapid increase in size will obliterate the urethral lumen and may cause difficulty in urination. It is very rare for this lesion to cause the complete closure of the urethral canal that leads to acute urinary retention.

\section{Case presentation}

A 21-year-old single man presented with acute urinary retention of one days' duration. The onset was sudden when he awoke in the morning. Urethral catheterization was performed in the Emergency Department and this drained $800 \mathrm{ml}$ of urine.

On further questioning, he claimed that he had had a painless lesion at the glans penis since childhood. The swelling increased in size in the past week causing discomfort. It was tolerable, and therefore he did not seek any medical treatment. He denied any other urinary symptoms or urethral discharge. He also denied any history of genital trauma or recent sexual intercourse.

On examination, we noted a small lesion over the ventral aspect of the glans penis, measuring about $3 \times 2 \mathrm{~cm}$ in size (Figure 1A). It was wellcircumscribed, bluish in color, but no punctum or signs of inflammation were seen. It was tender on palpation. Before the excision of the lesion, we removed the urethral catheter and re-examined the lesion. Without the catheter in situ, we noted that the urethral opening was completely obscured (Figure 1B). Thus, we postulated that he had developed urinary retention secondary to this lesion at the glans penis.

Our initial diagnosis was penile hemangioma complicated with thrombosis or intralesional rupture. Intraoperatively, we noted that the lesion was well-circumscribed and enclosed within a thin layer of capsule (Figure 1C). The urethral mucosa was not involved. We proceeded with excision of the lesion followed by meatoplasty. The postoperative period was uneventful and the urethral catheter was able to be removed on Day 3 after the operation. The histopathology result revealed a median raphe 
cyst of the penis with hemorrhagic features within the lesion (most likely caused by recent bleeding). During his first follow-up 6 weeks after the operation, uroflowmetry showed very good results with a peak flow of $30 \mathrm{ml} / \mathrm{s}$ and no significant residual urine in the urinary bladder.

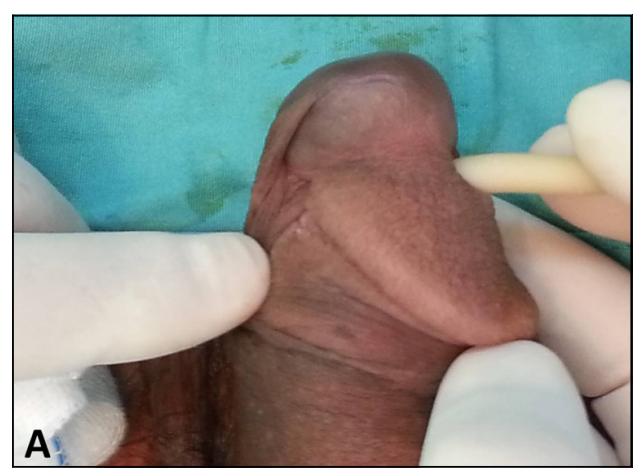

Figure 1A: A well-circumscribed small lesion over the ventral aspect of the glans penis, bluish in color, no signs of inflammation.

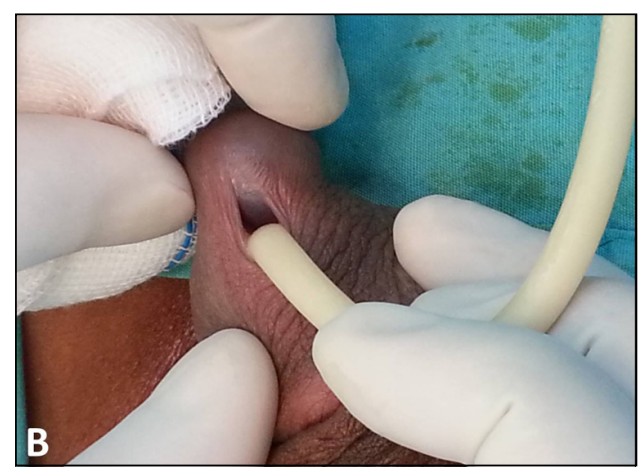

Figure 1B: The urethral opening was completely obscured by the lesion at the glans penis.

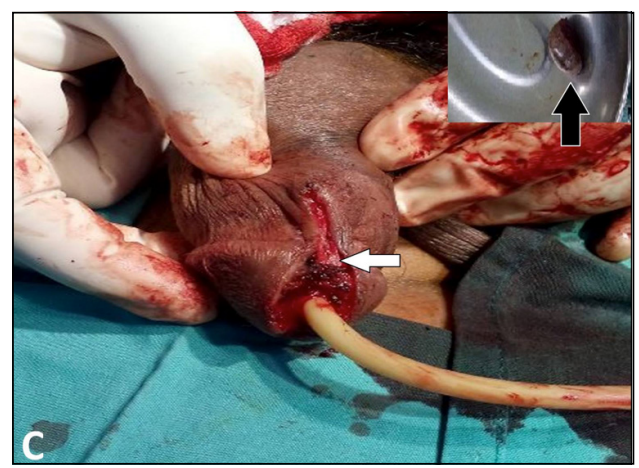

Figure 1C: The excised lesion was wellcircumscribed and enclosed within a thin layer of capsule (black arrow). Meatoplasty was done after the lesion was removed (white arrow).

\section{Discussion}

A median raphe cyst is an uncommon embryogenic developmental defect of the male genitalia. It was first described by Mermet in 1895. ${ }^{1}$ It is also known by several other names, including parameatal cyst, mucous cyst of the penis, and apocrine cystadenoma of the penile shaft. ${ }^{1}$ It can be found anywhere from the urethral meatus to the perineum and varies in size. The pathogenesis is postulated to be related to the fusion of the genitoperineal raphe at the midline. Therefore, the cyst development can be anywhere from the penis shaft to the perineum, with the most common site being at the penile shaft. ${ }^{2-4}$

The largest series of median raphe cysts were reported by Nishida et al. ${ }^{5}$ They reviewed 225 case reports from 1913 to 2010 and found that $83 \%$ of the cysts were solitary. ${ }^{5}$ There were six cases reported as pigmented median raphe cysts forming bluish discoloration of the lesion. ${ }^{5}$ The pigmented cysts were confirmed by the presence of melanin pigments in the histopathology analysis. ${ }^{5}$ In our case, the change in color was not caused by the presence of melanin pigment. Instead, the presence of hemorrhagic features in histology findings suggested that minor trauma could have caused the change in its color.

Median raphe cysts typically present as yellowish smooth papules that usually go unnoticed until trauma or infection occurs, giving rise to pain especially during erection or intercourse. ${ }^{1-4}$ In this case, the nodule mimicked a hemangioma, possibly due to the presence of intracystic hemorrhage. Yoshimoto et al. ${ }^{6}$ described a case of a blue-purple median raphe cyst of the penis that closely resembled our case, which was explained by the possibility of hemorrhage within the cyst. This patient had also received an initial diagnosis of hemangioma. ${ }^{6}$ However, a difference is that a history of zipper trauma occurring several years previously was apparent in the report by Yoshimoto et al. ${ }^{6}$ but not elicited in our case. We postulated that early morning erection possibly induced hemorrhagic changes in our case, as he denied a recent history of genitalia trauma or sexual intercourse.

The majority of penile median raphe cysts are asymptomatic and observation alone would suffice. However, with acute urinary retention as described above, intervention is needed to ease the symptoms. In this age group, the common causes of acute urinary retention include urethral stricture, urethral stone, and urethral trauma. ${ }^{7}$ The mainstay of treatment for a median raphe cyst is surgical excision. However, there is a variant of the condition which presents as elongated tracts known as 
canals. In this condition, videodermatoscopy is used in diagnosis. With a cystic lesion, simple removal would be sufficient, whereas the canal type needs to be excised fully to prevent a recurrence. ${ }^{4}$ To date, the best treatment option is surgical excision of the cyst as it gives the best cosmetic and functional outcome. ${ }^{2}$

\section{References}

1. Syed MMA, Amatya B, Sitaula S. Median raphe cyst of penis: a case report and review of the literature. J Med Case Rep. 2019;13:214223. doi:10.1186/s13256-019-2133-5

2. Alphones S, Phansalkar N, Manoharan P. Median raphe cyst of penis: A startling diagnosis for the unaccustomed clinician. Urol. Ann. 2019;11(3):314-316. doi:10.4103/ UA.UA_92_18

3. Ishida M, Iwai M, Yoshida K, Kagotani A, Okabe H. Pigmented median raphe cyst of the penis with consideration of the possible mechanism of melanocytic colonization: A case report. Oncol Lett. 2014;7(2):342-344. doi:10.3892/ol.2013.1719
4. LaCarrubba F, Tedeschi A, Francesconi L, Micali G. Canal versus cysts of the penile median raphe: advancing diagnostic methods using videodermatoscopy. Pediatric Dermatol. 2010;27(6):667-669. doi:10.1111/j.15251470.2010.01333.x

5. Nishida H, Kashima K, Daa T et al. Pigmented median raphe cyst of the penis. Cutan Pathol. 2012;39(8):808-810. doi:10.1111/j.1600-0560.2011.01862.x

6. Yoshimoto S, Ichinose M, Udagawa A, Matsumoto H, Shimizu S, Danino AM. Case of a rapidly growing blue-purple median raphe cyst of the penis. Ann of Plast Surg. 2002;48(1):108-109. doi:10.1097/00000637200201000-00021
7. Abdullahi M, Yunusa B, Mashi SA, Aji SA, Alhassan SU. Urinary retention in adults male patients: Causes and complications among patients manage in a teaching hospital in north western Nigeria. Open J Urology. 2016;6(7): 114-121. doi:10.4236/ oju.2016.67020. 


\section{Facial nerve palsy in otitis externa: A red flag?}

Aliyya Badaruddin, May May Choo

Badaruddin A, Choo MM. Facial nerve palsy in otitis externa: A red Flag?. Malays Fam Physician. 2021;16(1);117-120. https://doi.org/10.51866/cr1108

\section{Keywords:}

necrotizing otitis externa,

malignant otitis externa,

facial nerve palsy

\section{Authors:}

\section{Aliyya Badaruddin}

(Corresponding author)

MB, BCh, BAO, MOphthal

Department of Ophthalmology

University of Malaya Medical Centre

Kuala Lumpur, Malaysia

Email: aliyyabadaruddin@gmail.com

\section{Choo May May}

FRCS

Department of Ophthalmology University of Malaya Medical Centre Kuala Lumpur, Malaysia

\section{Abstract}

Otitis externa is an infection of the external auditory canal. It rarely results in facial palsy except in severe cases such as necrotizing otitis externa, which is a life-threatening invasive infection of the external auditory canal. Early recognition with prompt and appropriate treatment of necrotizing otitis externa is crucial to prevent more sinister complications. Here we report a case of an elderly gentleman who presented with otitis externa and developed facial palsy a month later. We identified possible problems that may have led to the complication so that such an occurrence can be prevented in the future.

\section{Introduction}

Facial palsy is an uncommon complication of otitis externa. It is more commonly seen in necrotizing otitis externa (NOE) with skull base osteomyelitis, especially if comorbidities are present. ${ }^{1}$ Here we report a case of an elderly diabetic patient who presented with otitis externa and subsequently developed facial palsy. We identified the possible problems that may have led to this complication.

\section{Case Report}

A 65-year-old man presented with right otalgia and otorrhoea for a duration of 2 days. He described the pain as excruciating and as sufficiently bad to disturb his sleep and disrupt some of his daily activities. He was diagnosed

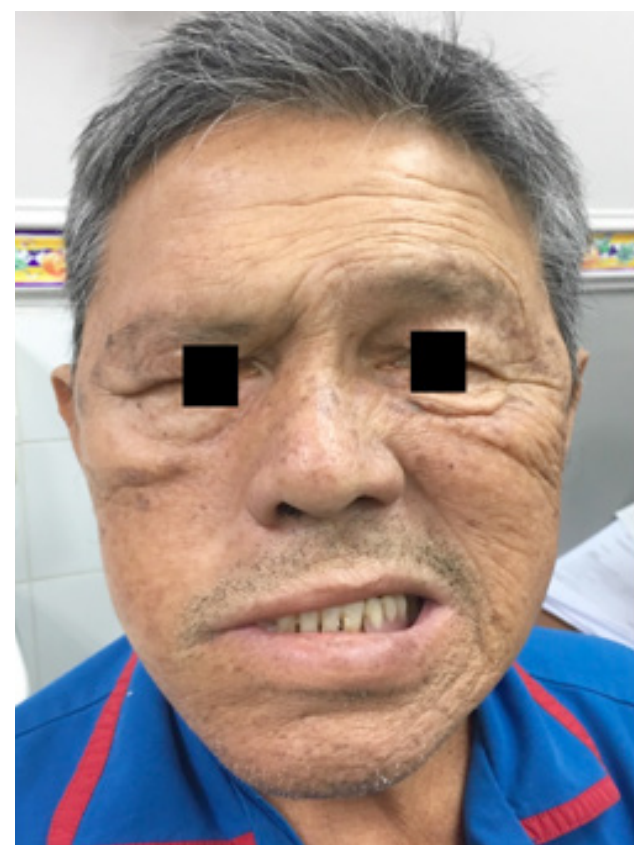

Figure 1: Right lower motor neurone facial palsy with right acute otitis externa by a general practitioner and completed 2 courses of oral antibiotics in addition to topical antibiotic. However, the pain did not resolve.

Four weeks later he developed facial asymmetry with drooling of saliva and was urgently referred to a neurologist to rule out a central cause. On examination, he had right lower motor neuron facial palsy with loss of right nasolabial fold, drooping of the corner of the forehead wrinkles (Figure 1).

$\mathrm{He}$ was then referred to the otorhinolaryngology team who discovered right severe otitis externa with granulation tissue in the external auditory canal. Right ear toileting and swab were performed, which

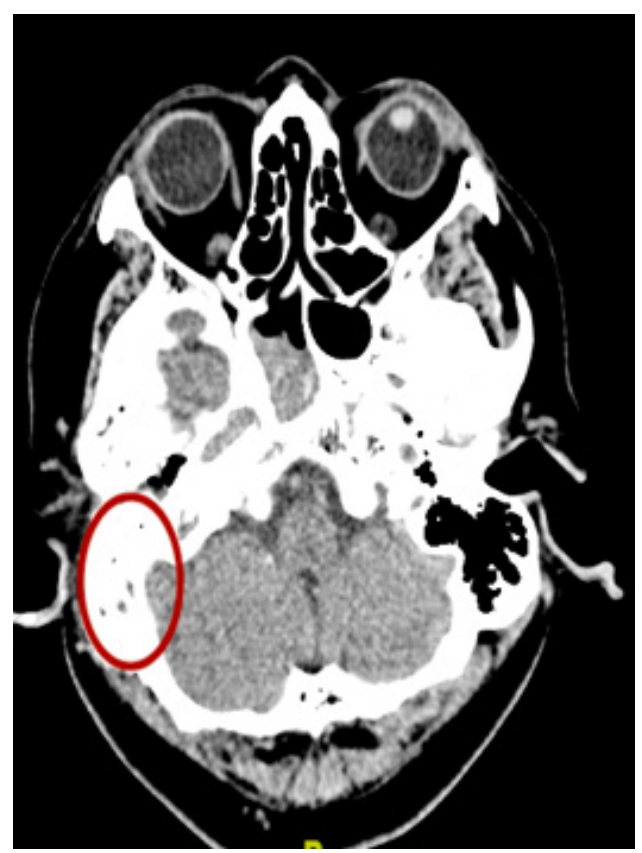

Figure 2: CT brain showing right mastoiditis and base of skull erosion mouth, right lagophthalmos and loss of right 
revealed Candida albicans. He was started on clotrimazole ear spray. He underwent High Resolution Computed Tomography of the temporal bone, which showed right mastoiditis and base of skull erosion (Figure 2). A biopsy ruled out malignant tumors. A subsequent Magnetic Resonance Imaging (MRI) showed enhancement at the right mastoid with no intracranial metastases (Figure 3). A final diagnosis of right necrotizing otitis externa with base of skull osteomyelitis and secondary facial palsy was made. He was started on intravenous ceftazidime $1 \mathrm{~g}$ every 8 hours, intravenous dexamethasone $8 \mathrm{mg}$ (in tapering doses) and underwent mastoidectomy (Figure 4).

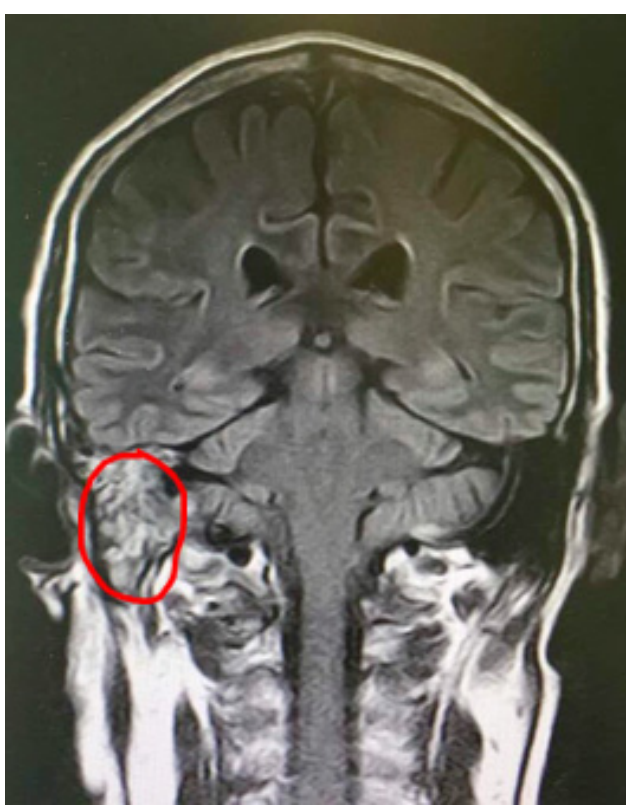

Figure 3: MRI brain/neck showing right mastoiditis

\section{Discussion}

Facial palsy is a rare complication of otitis externa. Previous studies have reported on facial palsy occurring in necrotizing otitis externa, especially in immunocompromised individuals such as diabetic patients. ${ }^{2,3}$ Diabetics have an increased $\mathrm{pH}$ in cerumen which predisposes them to infection such as Pseudomonas aeruginosa. The endarteritis and microangiopathy in diabetics leads to hypoperfusion in the subcutaneous tissue. ${ }^{1,4,5}$

Necrotizing otitis externa (NOE) associated with skull base osteomyelitis is an uncommon, rapidly spreading, life-threatening invasive infection of the external auditory canal (EAC) and the lateral skull base. ${ }^{1}$ Granulation tissue in the external auditory canal is a
Ophthalmic examination revealed right lagophthalmos with good Bell's reflex. There was mild punctate epithelial erosion. He was prescribed with lubricants and taping the right eye when asleep. The patient has had underlying diabetes for the past 8 years, which was not well-controlled with oral metformin $1 \mathrm{~g}$ and gliclazide $80 \mathrm{mg}$ twice daily. His random blood sugar was $13.0 \mathrm{mmol} / \mathrm{L}$ and $\mathrm{HbA} 1 \mathrm{C}$ $8 \%$. He was referred for optimization of sugar control while in the ward. Subcutaneous Insulatard $10 \mathrm{U}$ was added after which his blood sugar has been within normal range. There was gradual improvement of his facial palsy following rehabilitation including physiotherapy.

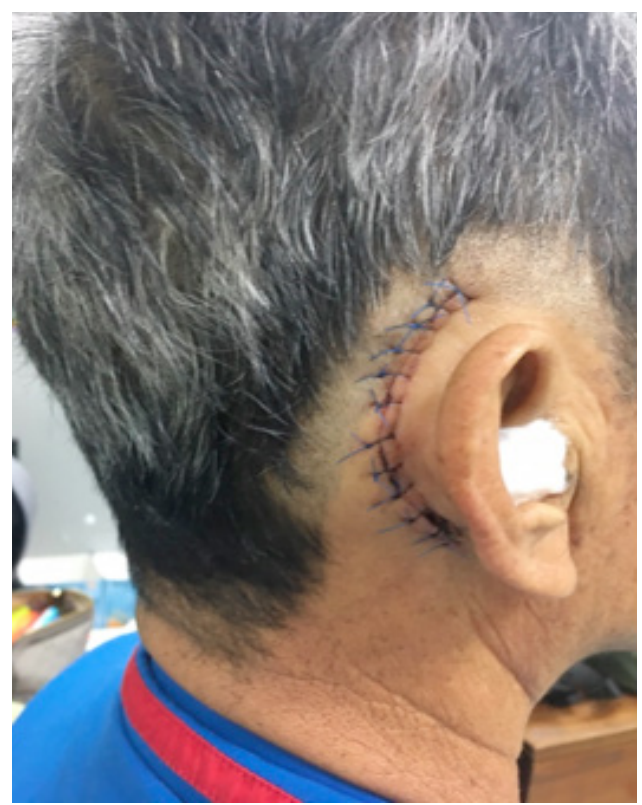

Figure 4: Right cortical mastoidectomy scar

pathognomonic finding of NOE. ${ }^{6}$ The disease may spread through the fissures of Santorini to the adjoining skull base, causing periostitis, osteomyelitis and cranial nerve palsies. ${ }^{1}$ It is very important to identify the disease and treat it early as it can cause more sinister complications such as meningitis, brain abscess and death. Treatment of NOE includes immunosuppression, local treatment of auditory canal and long-term antibiotic therapy (approximately 6-8 weeks). ${ }^{1,3}$ The role of surgery in NOE is limited; mastoidectomy is performed if mastoid air cells are involved and in persistent otalgia, a transection of $\mathrm{CN} \mathrm{V}$ and CN IX has been reported. ${ }^{3,7}$ Strict glycemic control is mandatory. ${ }^{3}$

Acute otitis externa (AOE) and NOE may have similar presentation in the beginning. However, 
in NOE the pain is so excruciating that it may disturb one's sleep and in later stages it can cause complications such as osteomyelitis of the skull with cranial nerves involvement or death from disseminated septic thrombo-emboli of the brain. Examination of NOE may show granulation tissue in EAC at the bone-cartilage junction. Imaging studies to confirm $\mathrm{NOE}$ include Computed Tomographic (CT) and bone scanning. Unlike AOE, NOE requires long-term intravenous antibiotic therapy. ${ }^{3,8}$ EAC cholesteatoma is an inflammatory lesion of the temporal bone with CT findings of soft tissue mass in EAC and adjacent bone erosion.'

There are several mechanisms thought to cause facial palsy in NOE. The primary mechanism is direct involvement of the nerve by toxins from the pathogens. Infection may spread through Santorini fissures to the base of the skull, causing bone destruction before spreading medially, thus triggering facial palsy. ${ }^{2,3}$ When facial palsy appears late in the disease, it may be due to silent mastoiditis, which may only be diagnosed with brain imaging. The presence of facial palsy indicates a poorer prognosis due to the deep penetration along the base of the skull. This may put other cranial nerve palsies at high risk and, due to the poor perfusion of the diseased area, a longer intravenous antibiotic treatment is required. ${ }^{3,10}$

Here we present a case of right facial palsy in a patient who was initially diagnosed with acute otitis externa which did not show improvement despite being treated with topical and oral antibiotics. We identified the possible causes that may have led to the complication of facial palsy. Firstly, when the patient initially presented with otalgia and otorrhoea, it is important to identify the causes and to start appropriate treatments. Ear secretions should be cultured prior to starting medications as early treatment with appropriate antimicrobials may achieve good cure rates and reduce the associated morbidity/mortality. ${ }^{1}$ Furthermore, the pattern of cultures may change after treatment has been initiated. ${ }^{3}$ If the infection is not responsive to antibiotics, a fungal infection should be considered. More importantly, early suspicion of NOE should be made if the symptoms remain persistent despite multiple courses of antibiotics. Hence, it is important to perform a good otoscopic examination and look for the presence of granulation tissue, especially in severe otalgia cases, for which a prompt referral to the otorhinolaryngology team should be made. ${ }^{1,10}$ Treating NOE with oral antibiotic alone as an outpatient is often inadequate; instead a long-term intravenous antibiotic is required. ${ }^{1,3}$

When patients with otitis externa went on to develop facial palsy, subsequent management of the problem may have been delayed by unnecessary referrals. This patient with lower motor neuron facial palsy associated with otalgia and otorrhoea was referred to the neurologist prior to seeing the otorhinolaryngology team, as it is crucial to exclude NOE. This, however, may have caused a delay in the treatment if the patient was not referred to the appropriate team promptly and may have resulted in more serious complications, including death. Therefore, a thorough examination must be performed to look for the causes of facial palsy in the ears, mastoid region, oral cavity, eyes, scalp, parotid glands and a complete neurological examination must be performed too before making a referral.

All patients with comorbidities including diabetes should be managed with a holistic approach. Treatment should not just focus on the otitis externa but also on any underlying health issues. The patient's sugar control should have been managed concurrently with the otitis when his case was first presented, as diabetic patients are more susceptible to a wide range of diseases including NOE. Medical experts should be involved earlier to co-manage any underlying comorbidities. In this case, if NOE had been diagnosed earlier during his initial presentation at the primary care level, the complication of facial nerve palsy may have possibly been avoided. A prompt referral and treatment in NOE cases may avoid other more serious complications including death.

\section{Conclusion}

Facial nerve palsy in otitis externa is an uncommon complication that may be prevented with proper management. This includes early recognition of the disease, early referrals, appropriate treatments and optimizing the comorbidities. Necrotizing otitis externa must be suspected in otitis externa cases in elderly diabetic patients who do not respond to oral antibiotic treatments.

\section{Funds Declaration}

No funds were received for this case report. 


\section{Patient Consent}

Written informed consent was obtained from the patient for this case report and its accompanying images.

\section{Disclosure of interest}

The authors report no conflicts of interest.

\section{How does this paper make a difference to general practice?}

- It creates awareness among clinicians and health staff of the need to recognize and differentiate necrotizing otitis externa (NOE) from acute otitis externa.

- It describes a case commonly encountered in a general-practice setting which may have been overlooked as a mild case but was ultimately discovered to be more complicated.

- A high index of suspicion for NOE is important when dealing with otitis externa not responding to topical/oral antibiotics.

\section{References}

1. Kumar SP, Ravikumar A, Somu L, Ismail NM. Malignant otitis externa: an emerging scourge. Journal of Clinical Gerontology and Geriatrics. 2013;4:128-131.

2. Walton J, Coulson C. Fungal malignant otitis externa with facial nerve palsy: tissue biopsy aids diagnosis. Case Rep Otolaryngol. 2014;2014:192318

3. Handzel O, Halperin D. Necrotizing (malignant) external otitis. Am Fam Physician. 2003; 68:309-312.

4. Singh A, Khabori M, Hyder J. Skull base osteomyelitis: diagnostic and therapeutic challenges in atypical presentation. Otolaryngol Head Neck Surg. 2005;133:121-125.
5. Illing E, Zolotar M, Ross E, Olaleye O, Molony N. Malignant otitis externa with skull base osteomyelitis. J Surg Case Rep. 2011;2011(5):6

6. Roberts JR. Malignant externa otitis: disastrous for diabetics. Emergency Medicine News. 2004;26:24-26.

7. Chandler JR. Pathogenesis and treatment of facial paralysis due to malignant external otitis. Ann Otol Rhinol Laryngol. 1972;81:648-658.

8. Cohen D, Friedman P, Filon A. Malignant external otitis versus acute external otitis. J Laryngol Otol. 1987;101: 211-215.
9. Heilbrun ME, Salzman KL, Glastonbury CM, et al. External auditory canal cholesteatoma: clinical and imaging spectrum. Am J Neuroradiol. 2003; 24: 751-756.

10. Cohen D, Friedman P. The diagnostic criteria of malignant external otitis. J Laryngol Otol. 1987;101:216-21. 